\title{
Analysis of Asymmetric Mutation Model in Random Local Search
}

\author{
Hiroshi Furutani* and Makoto Sakamoto \\ Faculty of Engineering, University of Miyazaki, Gakuen Kibanadai-nishi 1-1 \\ Miyazaki City, Miyazaki Prefecture, 889-2192, Japan \\ Yifei Du \\ Graduate School of Engineering, University of Miyazaki, Gakuen Kibanadai-nishi 1-1 \\ Miyazaki City, Miyazaki Prefecture, 889-2192, Japan \\ Kenji Aoki \\ Information Technology Center, University of Miyazaki, Gakuen Kibanadai-nishi 1-1 \\ Miyazaki City, Miyazaki Prefecture, 889-2192, Japan \\ E-mail: furutani@cs.miyazaki-u.ac.jp
}

\begin{abstract}
In a standard Evolutionary Algorithms (EAs), one uses the same rate for mutations from bit 1 to bit 0 and its reverse direction. There are many reports that the asymmetric mutation model is a very powerful strategy in EAs to obtain better solutions more efficiently. In this paper, we report stochastic behaviors of algorithms that are asymmetric mutation models of Random Local Search (RLS). The mathematical structure of asymmetry model can be derived in terms of a finite Markov chain. We demonstrate some useful results representing the effects of asymmetric mutation.
\end{abstract}

Keywords: Random Local Search, Asymmetric mutation, Hitting time, Markov chain

\section{Introduction}

Theoretical studies of EAs have been performed from various viewpoints. One of the most attractive objects of them is the convergence properties of EAs [1]. In the previous conference, we have reported a randomized heuristics, which mainly treated the computational complexity of Random Local Search (RLS) [2]. Our study used the results obtained in researches on Coupon Collector Problem (CCP), and made a mathematical analysis of hitting time in RLS by extending the original model of CCP.

In this conference, we report another extension of RLS, an asymmetric mutation model. We apply the asymmetric mutation in evolution of RLS; that is, $p_{a}$ for mutation $0 \rightarrow 1$ and $p_{b}$ for $1 \rightarrow 0$, respectively.
We carry out a theoretical analysis for the evolution of strings in the framework of a finite Markov chain [3].

The asymmetric mutation model is a very powerful strategy in EAs to obtain better solutions more efficiently [4]. In biology, spontaneous misreading of bases during DNA synthesis, mutation, is considered as a major factor contributing to evolution. Wada et al. showed that double-stranded DNA type strings can solve the knapsack problem effectively by using the asymmetric machinery of DNA replication [5]. They used different mutation rates for the leading and lagging DNA strands.

To analyze the behavior of the evolution processes, it is necessary to take into account of effects due to stochastic fluctuations. During the study of asymmetric mutation model of RLS, we noted mathematical papers 
in learning model with reinforcement, which gave the Markov chain model of learning processes [3]. We found that results in these papers can be interpreted as a model of RLS with asymmetric mutation rates, and apply these results in the present study.

In this model, we obtained an explicit form of Markov chain transition matrix, and furthermore the eigenvalues of this matrix were calculated by using rather technical methods. The largest eigenvalue is naturally $\lambda_{0}=1$, and the second largest one is $\lambda_{1}=1-\left(p_{a}+p_{b}\right) / l$. Since the most important factor to decide the speed of convergence is the second largest eigenvalue, we know the averaged value of two mutation rates mainly controls the speed of evolution. We will show in our report the behavior of solutions from the aspects of mathematical analysis and numerical simulations.

\section{Evolutionary Algorithms}

As a test function, we adopt OneMax function $f(x)$

$$
f(x)=\sum_{i=1}^{l} x_{i}, \quad x_{i} \in\{0,1\},
$$

where $x$ is a binary string of length $l$. We consider the maximization of OneMax function. The optimum solution is $x_{\text {opt }}=\{1\}^{l}$, and $f\left(x_{\text {opt }}\right)=l$.

1. The first choice of Evolutionary Algorithm is the Random Local Search (RLS). We define RLS as

\begin{tabular}{l} 
Algorithm $1 \quad$ Random Local Search \\
\hline 1: Initialize $x \in\{0,1\}^{l}$ uniformly at random. \\
2: Create $x^{\prime}$ by flipping one bit in $x$ which is \\
selected at random. \\
3: Select if $f\left(x^{\prime}\right)>f(x)$ then $x:=x^{\prime}$. \\
4: Go to 2 until a termination condition is fulfilled. \\
\hline 2. The next one is RLS with asymmetric mutatio \\
(ARLS). The ARLSis defined as \\
Algorithm $2 \quad$ RLS of Asymmetric Mutation \\
\hline 1: Initialize $x \in\{0,1\}^{l}$ uniformly at random. \\
2: Select one bit $x[j]$ in $x$ at random. \\
3: With probability $1-\left(p_{a}+p_{b}\right), x^{\prime}[j]=x[j]$. \\
If $x[j]=0$ then $x^{\prime}[j]=1$ with probability $p_{a}$. \\
If $x[j]=1$ then $x^{\prime}[j]=0$ with probability $p_{b}$. \\
4:If $x[j]$ is flipped then $x:=x^{\prime}$. \\
5: Go to 2 until a termination condition is fulfilled.
\end{tabular}

3. The third one is a lazy version of RLS, which is defined as

\begin{tabular}{l}
\hline Algorithm 3 Lazy RLS \\
\hline 1: Initialize $x \in\{0,1\}^{l}$ uniformly at random. \\
2: Select one bit $x[j]$ in $x$ at random. \\
3: Does not change $x[j]$ with probability $1-p_{a}$. \\
If $x[j]=0$ then $x^{\prime}[j]=1$ with probability $p_{a}$. \\
4: If $x[j]$ is flipped then $x:=x^{\prime}$. \\
5: Go to 2 until a termination condition is fulfilled.
\end{tabular}

This model is also defined as the variation of ARLS by putting $p_{b}=0$.

\section{Markov Chain Model}

This section presents the Markov chain approaches to the EAs. The search space of OneMax function is $\Omega=\{0,1\}^{l}$, and we divide $\Omega$ into $(l+1)$ subsets $\Omega=S_{0} \cup S_{1} \cup \cdots \cup S_{l}$, where $f\left(S_{i}\right)=i$.

\subsection{Asymmetric mutation model of RLS}

The transition matrix $P_{i, j}=P(j \mid i)$ represents the evolution of ARLS.

1. For $j=i+1,0 \leq i<l$

$$
P_{i, i+1}=p_{a}\left(1-\frac{i}{l}\right) \text {. }
$$

2. For $j=i-1,0<i \leq l$

$$
P_{i, i-1}=p_{a} \frac{i}{l}
$$

3. For $i=j, 0 \leq i \leq l$

$$
P_{i, i}=1-p_{a}\left(1-\frac{i}{l}\right)-p_{a} \frac{i}{l}
$$

For example, the transition matrix for $l=3$ is given by

$$
\boldsymbol{P}=\left(\begin{array}{cccc}
1-p_{a} & p_{a} & 0 & 0 \\
\frac{1}{3} p_{b} & 1-\frac{2}{3} p_{a}-\frac{1}{3} p_{b} & \frac{2}{3} p_{a} & 0 \\
0 & \frac{2}{3} p_{b} & 1-\frac{1}{3} p_{a}-\frac{2}{3} p_{b} & \frac{1}{3} p_{a} \\
0 & 0 & p_{b} & 1-p_{b}
\end{array}\right)
$$

The left eigenvectors $\boldsymbol{u}=\left(u_{0}, u_{1}, \cdots, u_{l}\right)$ satisfy, in the case of $l=3$,

$$
\begin{aligned}
\left(1-p_{a}\right) u_{0}+\frac{1}{3} p_{b} u_{1} & =\lambda u_{0}, \\
p_{a} u_{0}+\left(1-\frac{2}{3} p_{a}-\frac{1}{3} p_{b}\right) u_{1}+\frac{2}{3} p_{b} u_{2} & =\lambda u_{1}, \\
\frac{2}{3} p_{a} u_{1}+\left(1-\frac{1}{3} p_{a}-\frac{2}{3} p_{b}\right) u_{2}+p_{b} u_{3} & =\lambda u_{2}, \\
\frac{1}{3} p_{a} u_{2}+\left(1-p_{b}\right) u_{3} & =\lambda u_{3} .
\end{aligned}
$$

We define the $l$ th order polynomial function 


$$
f(z)=u_{0}+u_{1} z+u_{2} z^{2}+u_{3} z^{3}+\cdots+u_{l} z^{l} .
$$

Multiplying eigenvalue equations with $1, z, z^{2}, \ldots, z^{l}$, respectively, and summing them up, we have

$l\left(p_{a} z+1-p_{a}-\lambda\right) f(z)-(z-1)\left(p_{a} z+p_{b}\right) f^{\prime}(z)=0$.

Considering this as a differential equation of $f$, we have

$$
\begin{gathered}
f(z)=C(z-1)^{k}\left(p_{a} z+p_{b}\right)^{l-k}, \\
k=\frac{l(1-\lambda)}{p_{a}+p_{b}},
\end{gathered}
$$

where $C$ is an arbitrary constant. Since $f(z)$ is a polynomial of $l$ th order, $k$ must be an integer of $\{0,1, \ldots, l\}$. Then the eigenvalues are given by

$$
\lambda_{k}=1-\frac{k}{l}\left(p_{a}+p_{b}\right), \quad k=0,1, \ldots, l .
$$

The largest eigenvalue is $\lambda_{0}=1$, and the second largest one is $\lambda_{1}=1-\left(p_{a}+p_{b}\right) / l$, which determines the convergence speed of the chain.

The eigenvector corresponding to the largest eigenvalue $\lambda_{0}=1$ presents the distribution of the stationary state. In this case of $k=0$, we have

$$
f(z)=\left(p_{a} z+p_{b}\right)^{l}=\sum_{i=0}^{l}\left(\begin{array}{l}
l \\
i
\end{array}\right) p_{a}{ }^{i} p_{b}{ }^{l-i} z^{i},
$$

thus the components of eigenvector with normalization are given by

$$
u_{i}=\left(\begin{array}{l}
l \\
i
\end{array}\right) p_{a}{ }^{i} p_{b}{ }^{l-i} /\left(p_{a}+p_{b}\right)^{l}, \quad(0 \leq i \leq l) .
$$

From this, we can obtain the average number of bit ones,

$$
\bar{i}=l \frac{p_{a}}{p_{a}+p_{b}} .
$$

Similarly, we have the variance

$$
V(i)=l \frac{p_{a} \cdot p_{b}}{\left(p_{a}+p_{b}\right)^{2}} \text {. }
$$

Both quantities depend on the ratio of two mutation rates $p_{b} / p_{a}$.

\subsection{Lazy RLS}

The transition matrix for the lazy RLS shows that the Markov chain is absorbing one, and there are $l$ transient and one absorbing states, respectively. For absorbing Markov chains, the transition matrix is represented as

$$
\boldsymbol{P}=\left(\begin{array}{ll}
\boldsymbol{Q} & \boldsymbol{R} \\
\mathbf{0} & \boldsymbol{I}
\end{array}\right)
$$

The $l \times l$ submatrix $\boldsymbol{Q}$ shows transition probabilities among transient states $S_{0}, S_{1}, \cdots, S_{l-1}$. Since there is only one absorbing state, the unit matrix $\boldsymbol{I}$ is a scalar 1 .

For the calculation of the hitting time of the optimum solution, we use the fundamental matrix

$$
\boldsymbol{N}=(\boldsymbol{I}-\boldsymbol{Q})^{-1} \text {. }
$$

After some calculations, we have

$$
\begin{aligned}
N_{i, j} & =0, \quad(i>j) \\
& =\frac{l}{(l-j) p_{a}}, \quad(i \leq j) .
\end{aligned}
$$

The expected step $E_{i}(t)$ to enter into the absorbing state from the initial state $i$ is given by the vector $\boldsymbol{m}$ of $(l \times 1)$ [3]

$$
m=N 1,
$$

where $m_{i}$ is the expected step from ith state, and $\mathbf{1}$ is a column vector whose all entries are 1 .

The variance of $t$ is given by

$$
V(t)=(2 \boldsymbol{N}-\boldsymbol{I}) \boldsymbol{m}-\boldsymbol{m}^{2},
$$

where $\boldsymbol{m}^{2}$ is a column vector whose elements are $m_{i}{ }^{2}$.

\section{Numerical Experiment}

In this section, we demonstrate theoretical predictions and results of numerical calculations for comparison. We performed 10000 runs for each calculation, and obtained statistical quantities from them.

Figure 1 shows the stationary distribution of the asymmetric mutation model. The string length $\boldsymbol{l}=\mathbf{4 0}$, $\boldsymbol{p}_{\boldsymbol{a}}=\mathbf{0 . 0 3}$ and $\boldsymbol{p}_{\boldsymbol{b}}=\mathbf{0 . 0 2}$. The crosses are the result of numerical calculation. The solid line is the theoretical prediction obtained by eq.(2).

Figure 2 shows the distribution of the first hitting time of the optimum solution in Lazy RLS. The string length $\boldsymbol{l}=\mathbf{2 0}$ and mutation rate $p_{a}=\mathbf{0 . 3}$. The initial state is a bit string of all zeros. Dots are the numerical results. The solid line is the theoretical prediction obtained by using Markov chain transition matrix. 


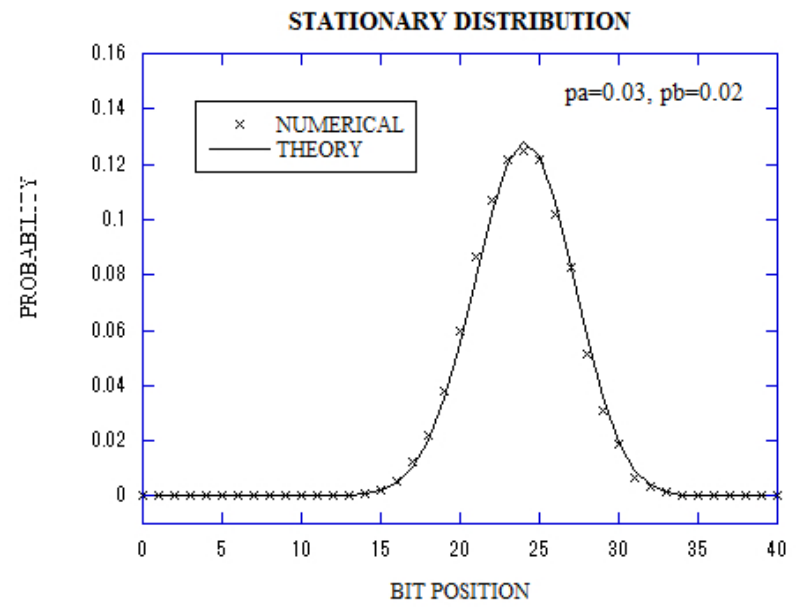

Fig. 1. Distribution of the stationary state in asymmetric mutation model of RLS with $\boldsymbol{l}=\mathbf{4 0}$. Mutation rates are $\boldsymbol{p}_{\boldsymbol{a}}=\mathbf{0 . 0 3}$ and $\boldsymbol{p}_{\boldsymbol{b}}=\mathbf{0 . 0 2}$, respectively.

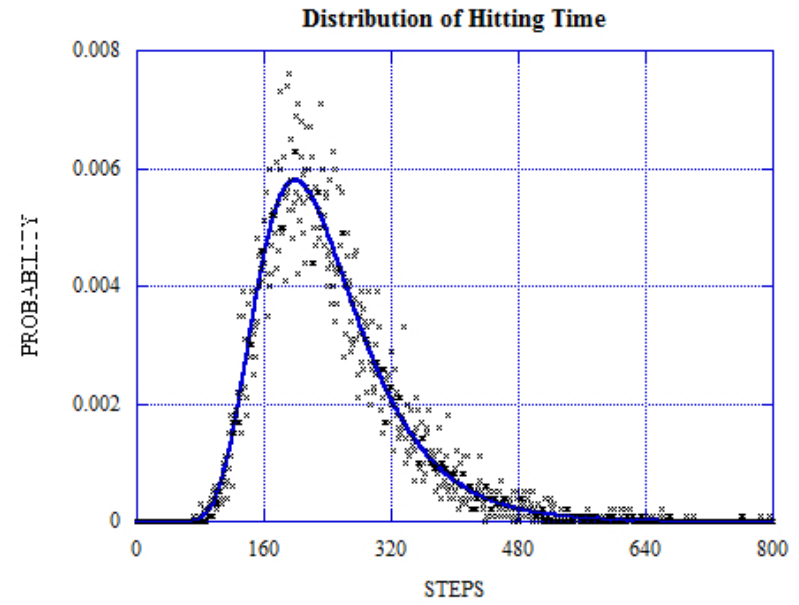

Fig. 2. Distribution of the first hitting time in Lazy RLS with $\boldsymbol{l}=\mathbf{2 0}$ and $\boldsymbol{p}_{\boldsymbol{a}}=\mathbf{0 . 3}$. The initial state is a bit string of all zeros. Dots are RLS calculations, and the solid line is the theoretical prediction.

Figure 3 shows the dependence of the average hitting time and its standard deviation (SD) on mutation rate $p_{a}$ in Lazy RLS. The string length $l$ is 20 . The initial state is a bit string of all zeros. The thin solid line is the theoretical prediction of the average hitting time, and the thick solid line represents the theoretical SD. Circles and crosses are numerical results.

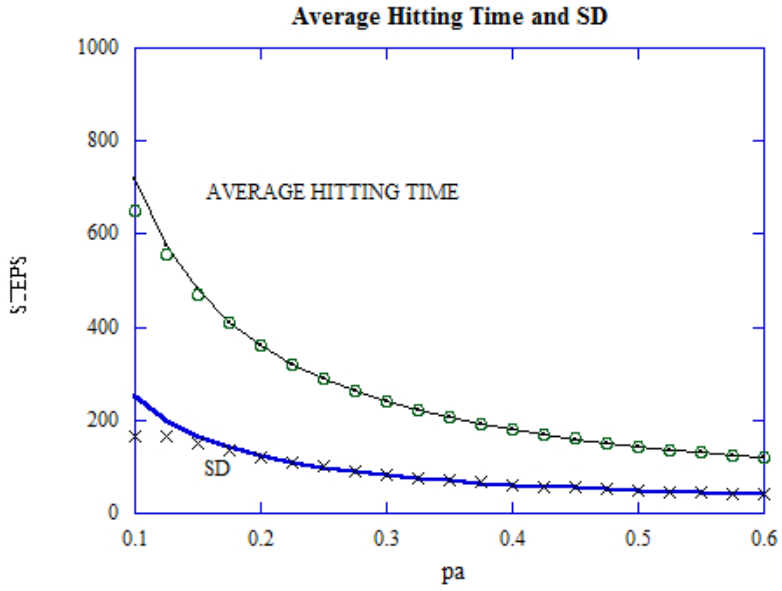

Fig. 3. Mutation rate dependence of the average hitting time and its standard deviation in Lazy RLS with $\boldsymbol{l}=\mathbf{2 0}$.

\section{Summary}

In this paper, we considered the behavior of the asymmetric mutation in the solving the optimization of OneMax function. This problem can be solved analytically in terms of a finite Markov chain [3]. We derived the eigenvalues and eigenvectors.

In genetic biology, it has been suggested by many statistical studies that asymmetric directional mutation pressures are commonly observed. It is interesting to study the phenomena using our theoretical approach.

\section{References}

1. P. S. Oliveto and X. Yao (2011), Runtime analysis of evolutionary algorithms for discrete optimization, in Theory of Randomized Search Heuristics, A. Auger and B. Doerr (eds), World Scientific, Singapore, pp.21--52.

2. H. Furutani, H. Tagami, Y. Du, and M. Sakamoto (2014), Markov chain analyses of Random Local Search and Evolutionary Algorithm, in Proceedings of the ICAROB2014.

3. M. Iosifescu (1980), Finite Markov chain processes and their applications, John Wiley \& Sons, New York.

4. B. Doerr, N. Hebbinghaus and F. Neumann (2007), Speeding up Evolutionary Algorithms through asymmetric mutation operator, Evolutionary Computation 15, pp.401--410.

5. K. Wada, H. Doi, S. Tanaka, Y. Wada and M. Furusawa (1993), A neo-Darwinian algorithm: Asymmetrical mutation due to semiconservative DNA-type replication promote evolution, Proceedings of National Academy of Science, USA 90, pp.11934--11938. 